\title{
RELACIÓN ENTRE LA GESTIÓN ADMINISTRATIVA DEL DIRECTOR Y EL MANTENIMIENTO Y MODERNIZACIÓN DE LA INFRAESTRUCTURA DE LAS INSTITUCIONES EDUCATIVAS PÚBLICAS DE LA RED Nº7 DE LA ZONA DE NUEVA ESPERANZA DE VILLA MARÍA DEL TRIUNFO, PROVINCIA Y REGIÓN LIMA, EN EL AÑO 2013.
}

RELATION BETWEEN THE ADMINISTRATIVE MANAGEMENT OF THE DIRECTOR AND THE MAINTENANCE AND MODERNIZATION OF THE INFRASTRUCTURE OF THE EDUCATIONAL PUBLIC INSTITUTIONS OF THE NETWORK N ${ }^{\circ} 07$ GIVES THE ZONE OF NEW HOPE OF VILLA MARIA OF THE VICTORY, PROVINCE AND REGION SMOOTHS, IN THE YEAR 2013.

Bach. NARVAEZ RETUERTO, Justo

\section{RESUMEN}

La investigación científica realizada es cuantitativa, básica, tiene un diseño correlacional causal, no experimental, los factores de estudio son dos: la Gestión Administrativa del Director y el mantenimiento y modernización de la infraestructura de las instituciones educativas públicas de la RED No 07 de la zona de Nueva Esperanza de Villa María del Triunfo, provincia y región Lima, en el año 2013. La población está conformada por 331 docentes y la muestra está conformada por 110 docentes.

Para la recolección de datos se confeccionaron dos cuestionarios: uno para la variable la Gestión Administrativa del Director y el otro para la variable el mantenimiento y modernización de la infraestructura, que se aplicó a los 110 docentes de la muestra.

Los instrumentos cumplen con las cualidades de validez y confiabilidad. El estudio plantea la siguiente hipótesis de investigación: Existe una relación significativa de la gestión del director y el mantenimiento y modernización de la infraestructura de las instituciones educativas públicas de la RED No 07 de la zona de Nueva Esperanza de Villa María del Triunfo, provincia y región Lima, en el año 2013.

Los resultados obtenidos nos permiten rechazar la hipótesis nula y aceptar la hipótesis investigada, según se aprecia los análisis estadísticos de los grupos de estudio, el nivel de significancia $\mathrm{p}=0,00$ menor que $\mathrm{p}=0,05(\mathrm{p}<\alpha)$. Se demuestra que el Rho de Spearman al ser 
0.835 tiene una correlación muy alta. Por lo tanto, se rechaza la hipótesis nula, comprobándose de este modo que: Existe una relación entre la gestión administrativa del director y el mantenimiento y modernización de la infraestructura de las instituciones educativas públicas de la RED No 07 de la zona de Nueva Esperanza de Villa María del Triunfo, provincia y región Lima, en el año 2013.

PALABRAS CLAVE: Gestión Administrativa del Director - El mantenimiento y modernización de la infraestructura.

\section{ABSTRACT}

The scientific realized investigation is quantitative, basic, has a design correlacional causal, not experimentally, the factors of study are two: the Administrative Management of the Director and the maintenance and modernization of the infrastructure of the educational public institutions of the NETWORK $\mathrm{N}^{\circ} 07$ of the zone of New Hope of Villa Maria of the Victory, province and region Lima, in the year 2013. The population is shaped by 331 teachers and the sample is shaped by 110 teachers.

For the compilation of information two questionnaires were made: one for the variable the Administrative Management of the Director and other one for the variable the maintenance and modernization of the infrastructure, which was applied to 110 teachers of the sample.

The instruments meet the qualities of validity and reliability. The study raises the following hypothesis: There is a significant relationship managing director and the maintenance and modernization of the infrastructure of public educational institutions No. 07 RED zone Nueva Esperanza Villa Maria del Triunfo, province and Lima region, in 2013.

The results allow us to reject the null hypothesis and accept the hypothesis investigated, according to statistical analysis of the study groups can be seen, the level of significance $p=$ $0.00 \mathrm{p}=$ less than $0.05(\mathrm{p}<\alpha)$. It is shown that Rho Spearman being 0.835 has a very high correlation. Therefore, the null hypothesis, proving thus is rejected: There is a relationship between the administration of the director and the maintenance and modernization of the infrastructure of public educational institutions No. 07 RED area New Hope Villa Maria del Triunfo, Lima province and region in 2013. 
KEYWORDS: Administrative Management Director - Maintenance and modernization of infrastructure.

\section{INTRODUCCIÓN}

Se puede observar cómo algunas instituciones educativas logran una mejora notable con una buena infraestructura y otras sin embargo se mantienen postradas sin un buen mantenimiento a pesar de que existen normas que respaldan la gestión de los recursos propios. Esto se puede evidenciar con los siguientes hechos: Servicios higiénicos en malas condiciones, aulas con las instalaciones eléctricas sin funcionar, paredes exteriores e interiores sin pintar, ventanas con vidrios rotos, $\mathrm{y}$ otros más con respecto al mantenimiento de las instalaciones de la institución educativa pública.

Con relación a la modernización de la infraestructura mencionamos que las aulas tienen mobiliario con más de veinte años de antigüedad, talleres con equipos y maquinarias que no funcionan, en algunos casos siguen utilizando máquinas de escribir, administración de documentos mecánica y anacrónica, ausencia de videotecas, entre otros.

Todas las Instituciones Educativas Públicas de la Educación Básica Regular (EBR), cuentan con ingresos propios o recursos financieros que pueden proceder de la expedición de certificados, alquileres de las instalaciones, expedición de constancias de estudio, pago de alquiler de los kioscos, cafetines, librerías y/o fotocopiadoras. Una adecuada gestión de estos recursos financieros debería verse reflejada en una adecuada infraestructura educacional, equipamiento, instalaciones y servicios educativos adecuados.

Según el Decreto $\mathrm{N}^{\mathrm{o}}$ 028-2007-ED "Reglamento de Gestión de Recursos Propios y Actividades Productivas y Empresariales en las Instituciones Educativas Públicas" en el Artículo $2^{\circ}$, señala que el objetivo es fortalecer la gestión de las instituciones educativas, contribuyendo a su auto sostenimiento y desarrollo institucional. La Gestión de recursos involucra a las actividades de conducción de los recursos humanos, materiales, financieros, tecnológicos y de tiempo que constituyen medios para lograr los propósitos educativos. La presente investigación determinará la influencia que tiene una adecuada gestión de los recursos propios en el mantenimiento $\mathrm{y}$ modernización de la infraestructura en las instituciones educativas públicas.

$\begin{array}{lllll}\text { El Congreso de } & \text { la } & \text { República en } \\ \text { coordinación con el } & \text { Ministerio de }\end{array}$


Economía y Finanzas y el Ministerio de Educación han implementado el "Programa de Mantenimiento de Locales Escolares" según la Ley No 29142, Ley de Presupuesto del Sector Público Ejercicio Fiscal 2008, lo que significaría que las Instituciones Educativas Públicas recibirán por primera vez y directamente del Estado los recursos Financieros del Tesoro Público durante el mes de enero, este mantenimiento de Locales Escolares consiste en un conjunto de acciones que se deben realizar para conservar en buenas condiciones el local escolar y sus instalaciones.

Estas actividades se dividen en: mantenimiento preventivo $\mathrm{y}$ mantenimiento correctivo. Los recursos pueden priorizarse en la reparación de instalaciones sanitarias, letrinas, lavatorios, griferías, instalaciones eléctricas, mobiliario (mesas, sillas, pizarras, etc.).

A partir de la afirmación que: "las escuelas eficientes están abiertas a la innovación en busca de mejores respuestas a los problemas comunes que se suceden" GATHER (2001) y que un Centro Educativo puede convertirse en un punto estratégico para la innovación educativa, Gather (2001) plantea que una de las dimensiones del funcionamiento de la escuela que se debe trabajar es la organización del mismo, se analiza algunos estudios sobre organización.

Para ÁLVAREZ (2001), todos los modelos de gestión de calidad: modelo Deming (Japón), modelo Baldrige (EE.UU.), modelo EFQM (Europa), etc., consideran el ejercicio de un cierto tipo de liderazgo como un factor básico de la calidad. De acuerdo con Jiménez (2001), nos interesa subrayar, respetando las diferencias entre gestión y liderazgo, el estrecho paralelismo y lógica compatibilidad que puede establecerse entre las propuestas generadas a partir de la gestión de calidad total con las comunidades de liderazgo propuestas para las organizaciones que aprenden y una buena infraestructura y su mantenimiento.

TORRES (2000, P. 6) hace referencia al término innovación relacionándolo con el de reforma. Reserva el de innovación para las intervenciones que tienen lugar "abajo", a nivel micro/local, dentro o fuera del sistema escolar, mientras que el de reforma lo asigna a las intervenciones "desde arriba", a nivel macro y de sistema (retomaremos este aspecto cuando hablemos de reforma).

$\mathrm{Y}$ todos los seres humanos tienen capacidad para adelantar. Esto implica una estructura que valorice a todas las personas de la empresa. Es tan sencillo como eso." En palabras de DRUCKER (2000) “el 
liderazgo tiene que surgir de la responsabilidad; tiene que estar sujeto a la rendición de cuentas. El buen liderazgo no lo demuestran los logros de líder, sino lo que pasa después de que éste abandona el escenario.

El liderazgo es hacer cosas, Álvarez (2001) afirma que la calidad de las escuelas depende en gran medida de la competencia, dedicación y estilo de liderazgo del equipo directivo. Pero, si bien es cierta la coincidencia, cada vez mayor, al destacar la dirección como requisito necesario el desarrollo de procesos educativos eficaces, también lo es la ausencia de consenso sobre el modo de organizar y ejercer esa dirección. No es posible establecer un estilo ideal de liderazgo o director, ya que la actuación ha de adaptarse a muchas variables y contingencias.

\section{Según HARGREAVES (2003, P. 26-36)}

las escuelas enfrentan problemas que deben atender y que se originan en su necesidad de establecer conexiones conscientes y constructivas con el mundo que las trasciende, por varias razones:

1. Como nunca antes, las escuelas no pueden cerrar sus puertas y dejar fuera los problemas del mundo exterior

2. Las escuelas están perdiendo su monopolio sobre el aprendizaje.
3. En muchas partes del mundo desarrollado las personas experimentan una crisis de comunidad, y las escuelas brindan una de nuestras últimas y mayores esperanzas de resolverla.

4. Los docentes necesitan mucha más ayuda.

5. La competencia en el mercado, la elección parental y la autogestión individual, redefinen las relaciones de las escuelas con sus entornos.

6. Las escuelas ya no pueden ser indiferentes a la vida laboral que espera a sus alumnos cuando ingresen en el mundo adulto.

Al referirse a la innovación, (NEIROTTI \& POGGI, 2004, P. 168 Y SS.) consigna que el mismo concepto plantea un problema; como también la falta de un marco teórico suficientemente desarrollado y compartido que permita decir qué es innovador y qué no. Entre las dificultades que se encuentran para caracterizar una innovación educativa, consigna que se trata de un concepto altamente relativo, pues una innovación no es aséptica ni neutra, pues está condicionada por posicionamientos políticos, sociales, culturales y epistemológicos, de tal forma que lo que es innovador para una persona o grupo, no lo es para otros. 
En la tesis presentada por (CÁCERES

CASAVERDE, 2008) señala que la teoría neoclásica otorga un modelo para este tipo de investigación pues aporta posibles soluciones para mejorar el servicio que brindan las instituciones educativas. Asimismo, señala lo siguiente: la administración es planear, organizar, dirigir, controlar, y son principios generales. Los gerentes o directores son necesarios para cualquier institución dinámica y exitosa porque deben planear, dirigir y controlar los procesos de los servicios que brindan.

CARBONELL (2001, P. 18) aclara que la mera modernización de la escuela nada tiene que ver con la innovación (introducción de ordenadores, prácticas de huerta, etc.) si no se modifican las concepciones sobre la enseñanza y el aprendizaje. En este trabajo sobre modernización de la IIEE, queremos resaltar que siendo un tema de gestión de liderazgo del director se convierte en un tema importante para la enseñanzaaprendizaje a partir de la noción clara de provisión de los insumos que hacen eficaz los logros de aprendizaje.

\section{Para IDALBERTO CHIAVENATO}

(2007), "una organización es un sistema de actividades conscientemente coordinadas de dos o más personas”. La cooperación entre estas personas es esencial para la existencia de la organización. Una organización existe solo cuando hay personas capaces de comunicarse, que están dispuestas a contribuir con una acción conjunta a fin de alcanzar un objetivo común" (Chiavenato, 2007). Cuán importante entonces, identificar de qué formas estos aspectos esenciales que le dan sentido a una organización, se cumplen en el caso de las entidades educativas. En segundo lugar, está la distinción que hace el mismo Chiavenato (2007) entre eficiencia y eficacia, señalando que la primera "es una medida normativa de la utilización de los recursos en los procesos" (deber hacer) mientras que la segunda "es una medida normativa de logro de resultados" (deber alcanzar). En particular, la juventud, los profesores y profesoras recientemente graduados, pueden ser una fuente de agitación o malestar, aunque no tengan un acceso inmediato a los canales de la influencia política (1989, p. 73).

Una de las conclusiones a las que MACMILLAN (2000, P. 68) arriba en su estudio al igual que otros es la de que el poder de la cultura de los docentes es un gran promotor o un gran obstáculo para el cambio educativo.

OBJETIVOS

DE

LA

INVESTIGACIÓN

\section{Objetivo General}


Determinar la relación entre la gestión del director y el mantenimiento y modernización de la infraestructura de las instituciones educativas públicas de la RED No 07 de la zona de Nueva Esperanza de Villa María del Triunfo, Provincia de Lima, Región Lima, 2013.

\section{Objetivos Específicos}

1. Identificar la relación que existe entre la Gestión del Director y el mantenimiento de la infraestructura de las instituciones educativas públicas de la RED No 07 de la zona de Nueva Esperanza de Villa María del Triunfo, provincia y región Lima, en el año 2013.

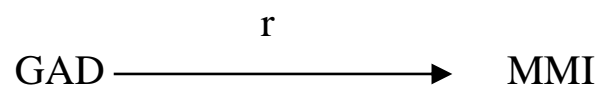

2. Determinar la relación entre la Gestión del Director y la modernización de la infraestructura de las instituciones educativas públicas de la RED Nº7 de la zona de Nueva Esperanza de Villa María del Triunfo, provincia y región Lima, en el año 2013.

\section{MATERIAL Y MÉTODO:}

El estudio tiene dos variables; la variable Y (Mantenimiento y modernización de la infraestructura de las instituciones educativas) es el efecto y la causa es la variable X (Gestión Administrativa del Director). El diseño es un correlacional causal, el cual permitirá establecer la relación entre las variables del estudio, se representa de la siguiente manera:

\footnotetext{
GAD $(\mathrm{X}) \quad=\quad$ Estilo de Liderazgo Directivo

MMI $(\mathrm{Y})=$ Mantenimiento $\mathrm{y}$ modernización de la infraestructura de las instituciones educativas

$\mathrm{r} \quad=\quad$ Correlación
} 


\section{RESULTADOS Y DISCUSION:}

Luego de realizar el levantamiento de la información a través de las 2 encuestas, encontramos los siguientes resultados:

TABLA $\mathbf{N}^{\circ} \mathbf{1}$

Contingencia de la Variable Mantenimiento y Modernización de la Infraestructura

\begin{tabular}{|c|c|r|r|r|r|}
\hline \multicolumn{2}{|c|}{} & Frecuencia & Porcentaje & $\begin{array}{l}\text { Porcentaje } \\
\text { válido }\end{array}$ & $\begin{array}{c}\text { Porcentaje } \\
\text { acumulado }\end{array}$ \\
\hline \multirow{3}{*}{ Válidos } & Bajo & 4 & 3,6 & 3,6 & 3,6 \\
\cline { 2 - 6 } & Medio & 104 & 94,5 & 94,5 & 98,2 \\
\cline { 2 - 6 } & Alto & 2 & 1,8 & 1,8 & 100,0 \\
\cline { 2 - 6 } & Total & 110 & 100,0 & 100,0 & \\
\hline
\end{tabular}

Fuente: el autor.

\section{FIGURA $\mathbf{N}^{\circ} 1$ \\ Barras del Mantenimiento y Modernización de La Infraestructura}

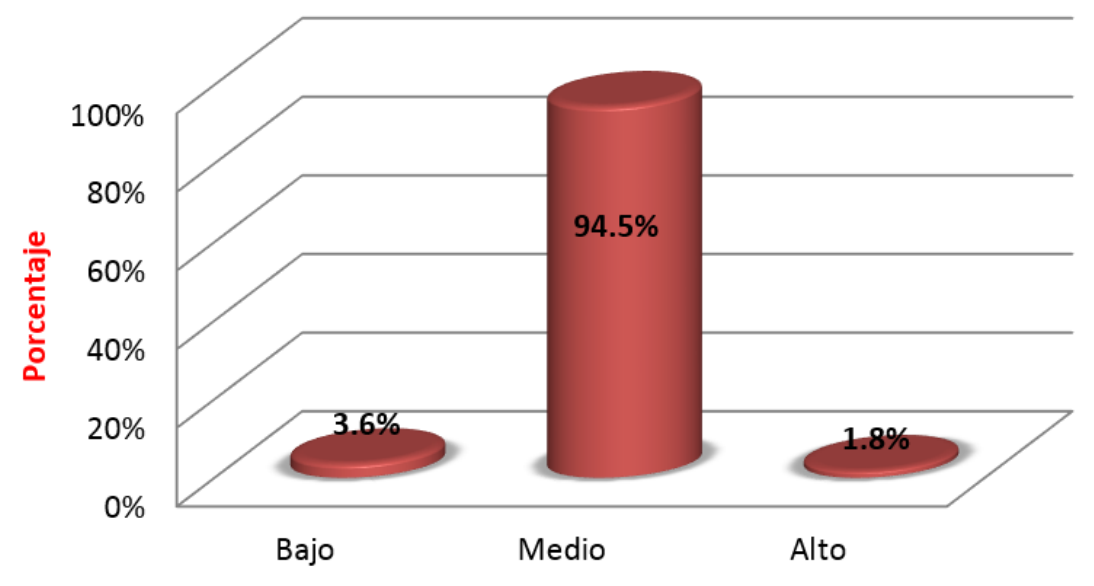

Fuente: el autor.

Observamos que se presenta mayormente un nivel medio en el mantenimiento y modernización de la infraestructura de las instituciones educativas públicas de la RED No 07 de la zona de Nueva Esperanza de Villa María del Triunfo, provincia y región Lima, en el año 2013.”; es decir el $94,5 \%$ presenta un nivel medio de mantenimiento y modernización. El 3.6\% presenta un nivel bajo en mantenimiento y modernización. El $1.8 \%$ presenta un nivel de mantenimiento y modernización alto. Estos resultados nos indican la mayoría de instituciones educativas de la RED No7 de la zona de Nueva Esperanza presentan un nivel medio. 
TABLA $\mathbf{N}^{\circ} \mathbf{2}$

Variable Gestión Administrativa

\begin{tabular}{|r|c|r|r|r|r|}
\hline \multicolumn{2}{|c|}{} & Frecuencia & Porcentaje & \multicolumn{1}{l|}{$\begin{array}{l}\text { Porcentaje } \\
\text { válido }\end{array}$} & $\begin{array}{l}\text { Porcentaje } \\
\text { acumulado }\end{array}$ \\
\hline \multirow{4}{*}{ Válidos } & Bajo & 5 & 4,5 & 4,5 & 4,5 \\
\cline { 2 - 6 } & Medio & 104 & 94,5 & 94,5 & 99,1 \\
\cline { 2 - 6 } & Alto & 1 & 9 &, 9 & 100,0 \\
\cline { 2 - 6 } & Total & 110 & 100,0 & 100,0 & \\
\hline
\end{tabular}

Fuente: el autor.

FIGURA $\mathbf{N}^{\circ} 2$

Barras de Gestión Administrativa

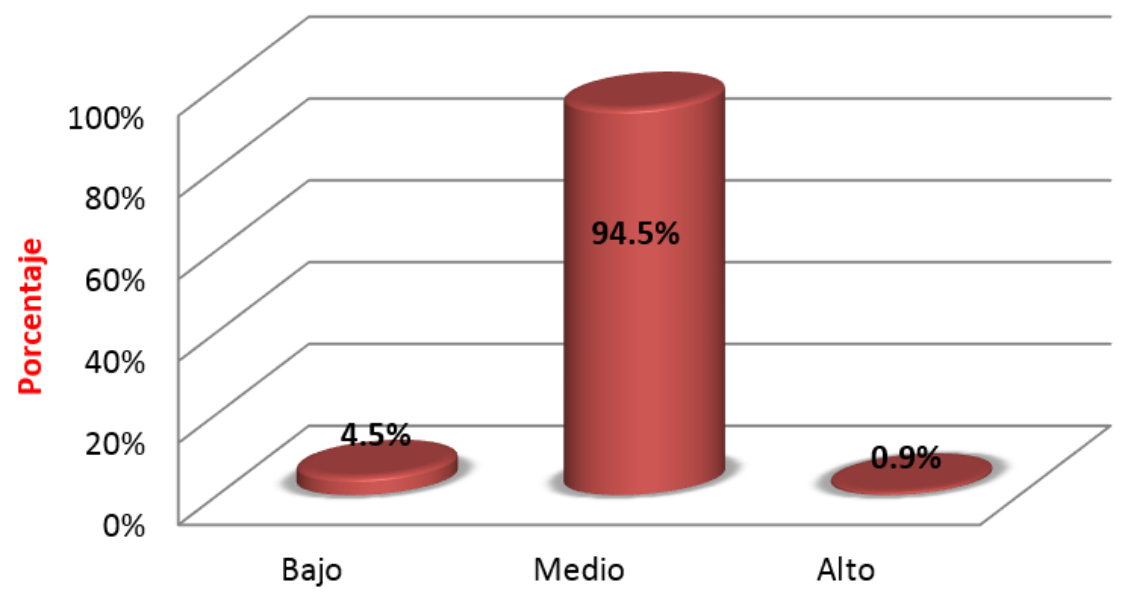

Fuente: El autor

Observamos que la mayoría de instituciones poseen un nivel medio en la Gestión Administrativa en las instituciones educativas públicas de la RED No 07 de la zona de Nueva Esperanza de Villa María del Triunfo, provincia y región Lima, en el año 2013.”; es decir el 94,5\% presenta un nivel medio de Gestión Administrativa. El
4.5\% presenta un nivel bajo en Gestión Administrativa. El $0.9 \%$ presenta un nivel en Gestión Administrativa alto. Estos resultados nos indican que la mayoría de instituciones educativas de la RED N ${ }^{\circ} 7$ de la zona de Nueva Esperanza presentan una Gestión Administrativa de nivel medio. 
TABLA $\mathbf{N}^{\circ} \mathbf{3}$

Contingencia entre el Mantenimiento y Modernización de la Infraestructura

\begin{tabular}{|c|c|c|c|c|c|}
\hline \multicolumn{2}{|c|}{} & Frecuencia & Porcentaje & $\begin{array}{c}\text { Porcentaje } \\
\text { válido }\end{array}$ & $\begin{array}{c}\text { Porcentaje } \\
\text { acumulado }\end{array}$ \\
\hline \multirow{4}{*}{ Válidos } & Bajo & 6 & 5,5 & 5,5 & 5,5 \\
\cline { 2 - 6 } & Medio & 40 & 36,4 & 36,4 & 41,8 \\
\cline { 2 - 6 } & Alto & 64 & 58,2 & 58,2 & 100,0 \\
\cline { 2 - 6 } & Total & 110 & 100,0 & 100,0 & \\
\hline
\end{tabular}

Fuente: El autor

FIGURA $\mathbf{N}^{\circ} 3$

Barras del Mantenimiento y Equipamiento

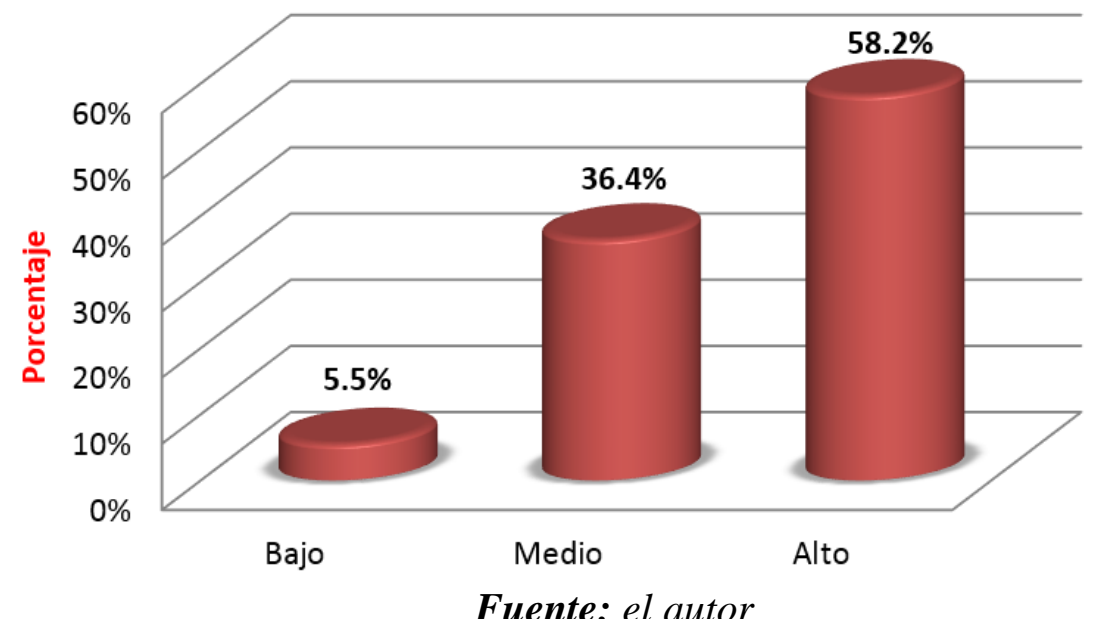

Fuente: el autor

Observamos que se presenta mayormente un nivel alto en el mantenimiento y equipamiento de la infraestructura de las instituciones educativas públicas de la RED No 07 de la zona de Nueva Esperanza de Villa María del Triunfo, provincia y región Lima, en el año 2013.”; es decir el $58.2 \%$ presenta un nivel alto de mantenimiento y equipamiento. El $36.4 \%$ presenta un nivel medio en mantenimiento y equipamiento. El 5.5\% presenta un nivel de mantenimiento y equipamiento bajo. Estos resultados nos indican la mayoría de instituciones educativas de la RED N7 de la zona de Nueva Esperanza presentan un nivel alto. 
TABLA $\mathbf{N}^{\circ} \mathbf{4}$

Contingencia Modernización de la Infraestructura

\begin{tabular}{|c|c|c|c|c|c|}
\hline & & Frecuencia & Porcentaje & $\begin{array}{l}\text { Porcentaje } \\
\text { válido }\end{array}$ & $\begin{array}{l}\text { Porcentaje } \\
\text { acumulado }\end{array}$ \\
\hline \multirow{4}{*}{ Válidos } & Bajo & 20 & 18,2 & 18,2 & 18,2 \\
\hline & Medio & 73 & 66,4 & 66,4 & 84,5 \\
\hline & Alto & 17 & 15,5 & 15,5 & 100,0 \\
\hline & Total & 110 & 100,0 & 100,0 & \\
\hline
\end{tabular}

Fuente: El autor

FIGURA $\mathbf{N}^{\circ} 4$

Barras de Modernización de la Infraestructura

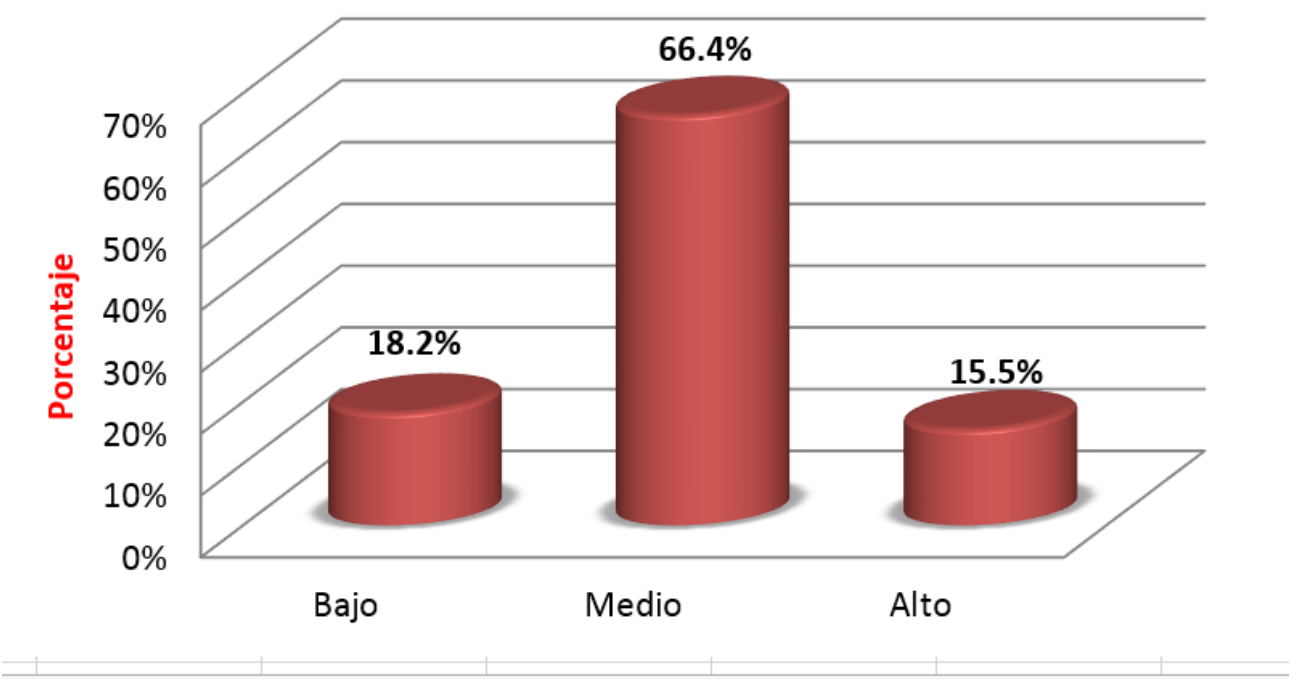

Fuente: el autor.

Observamos que se presenta mayormente un nivel medio en la modernización de la infraestructura de las instituciones educativas públicas de la RED No 07 de la zona de Nueva Esperanza de Villa María del Triunfo, provincia y región Lima, en el año 2013.”; es decir, el $66.4 \%$ presenta un nivel medio de modernización. El 18.2\% presenta un nivel bajo en modernización. El $15.5 \%$ presenta un nivel de modernización alto. Estos resultados nos indican que la mayoría de instituciones educativas de la RED $\mathrm{N}^{\circ} 7$ de la zona de Nueva Esperanza, presentan un nivel medio en la modernización de la infraestructura. 
TABLA $\mathbf{N}^{\circ} \mathbf{5}$

Contingencia Modernización de la Maquinaria

\begin{tabular}{|c|c|c|c|c|c|}
\hline \multicolumn{2}{|c|}{} & Frecuencia & Porcentaje & $\begin{array}{c}\text { Porcentaje } \\
\text { válido }\end{array}$ & $\begin{array}{c}\text { Porcentaje } \\
\text { acumulado }\end{array}$ \\
\hline \multirow{4}{*}{ Válidos } & Bajo & 10 & 9,1 & 9,1 & 9,1 \\
\cline { 2 - 6 } & Medio & 91 & 82,7 & 82,7 & 91,8 \\
\cline { 2 - 6 } & Alto & 9 & 8,2 & 8,2 & 100,0 \\
\cline { 2 - 6 } & Total & 110 & 100,0 & 100,0 & \\
\hline
\end{tabular}

Fuente: el autor.

FIGURA $\mathbf{N}^{\circ} 5$

Barras de la Modernización de la Maquinaria Escolar

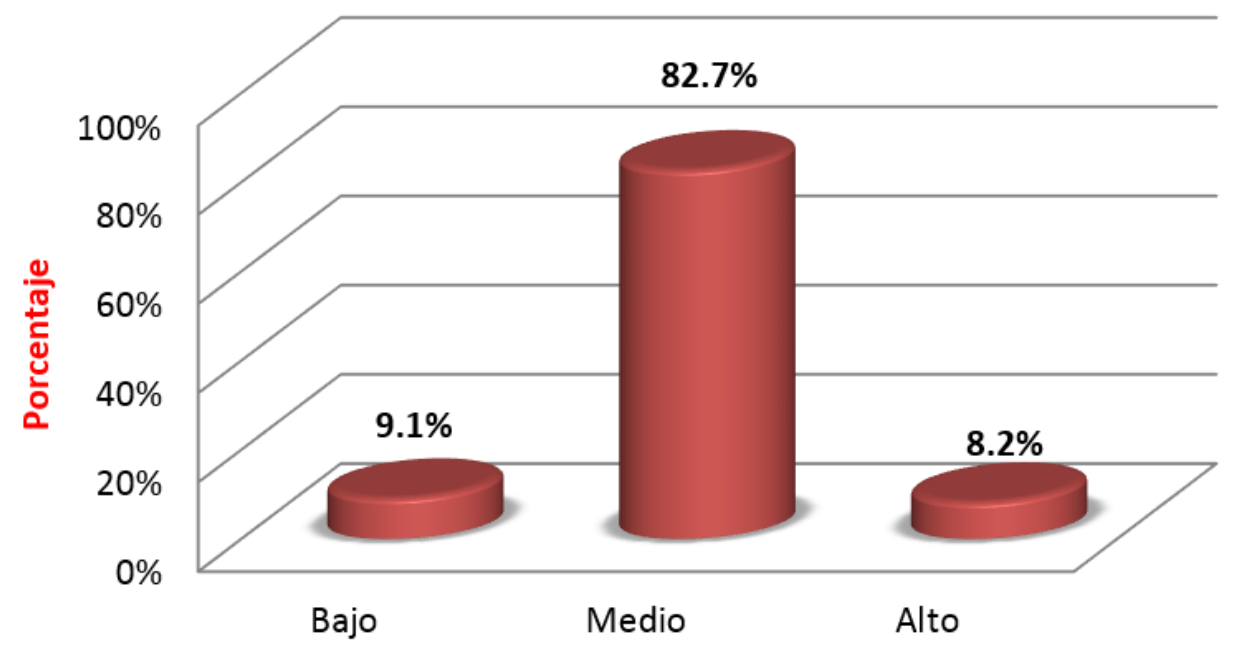

Fuente: el autor

Observamos que se presenta mayormente un nivel medio en la modernización de la maquinaria de las instituciones educativas públicas de la RED No 07 de la zona de Nueva Esperanza de Villa María del Triunfo, provincia y región Lima, en el año 2013."; es decir el $82.7 \%$ presenta un nivel medio de modernización de la maquinaria. El $9.1 \%$ presenta un nivel bajo en modernización de la maquinaria. El 8.2\% presenta un nivel de modernización de la maquinaria, alto.

\section{CONCLUSIONES}

PRIMERA. - Se demuestra que existe relación entre la gestión administrativa del director con el mantenimiento y modernización de la infraestructura de las instituciones educativas públicas de la RED No 07 de la zona de Nueva Esperanza de Villa María del Triunfo, provincia y región Lima, en el año 2013, según la prueba de Rho Spearman, se aprecia en los estadísticos de los grupos de estudio, siendo el nivel de significancia $\mathrm{p}=0,000$ menor que $\mathrm{p}=0,05(\mathrm{p}<\alpha)$. 
SEGUNDA. - Se demuestra que existe relación entre la Gestión Administrativa del Director con el mantenimiento y equipamiento de la infraestructura de las instituciones educativas públicas de la RED N 07 de la zona de Nueva Esperanza de Villa María del Triunfo, provincia y región Lima, en el año 2013, según la prueba de Rho Spearman, se aprecia en los estadísticos de los grupos de estudio, siendo el nivel de significancia $\mathrm{p}=0,000$ menor que $\mathrm{p}=0,05(\mathrm{p}<\alpha)$.

TERCERA. - Se demuestra que existe relación entre la Gestión Administrativa del Director con la modernización de la infraestructura de las instituciones educativas públicas de la RED No 07 de la zona de Nueva Esperanza de Villa María del Triunfo, provincia y región Lima, en el año 2013, según la prueba de Rho Spearman, se aprecia en los estadísticos de los grupos de estudio, siendo el nivel de significancia $\mathrm{p}=0,000$ menor que $\mathrm{p}=0,05$ $(\mathrm{p}<\alpha)$.

CUARTA. - Se demuestra que existe relación entre la Gestión Administrativa del Director con la modernización de la maquinaria de la infraestructura de las instituciones educativas públicas de la RED No 07 de la zona de Nueva Esperanza de Villa María del Triunfo, provincia y región Lima, en el año 2013, según la prueba de Rho Spearman, se aprecia en los estadísticos de los grupos de estudio, siendo el nivel de significancia $\mathrm{p}=0,000$ menor que $\mathrm{p}=0,05$ ( $\mathrm{p}<$ alfa).

\section{RECOMENDACIONES}

PRIMERA. - Se recomienda que se capacite a los directores en gestión administrativa para obtener mejores resultados en el mantenimiento $\mathrm{y}$ modernización de la infraestructura de las instituciones educativas públicas de la RED No 07 de la zona de Nueva Esperanza de Villa María del Triunfo, provincia y región Lima.

SEGUNDA. - Se recomienda que se mejore la gestión administrativa del director para obtener mejores resultados en el mantenimiento y equipamiento de la infraestructura de las instituciones educativas públicas de la RED No 07 de la zona de Nueva Esperanza de Villa María del Triunfo, provincia y región Lima.

TERCERA. - Se recomienda que se mejore la gestión administrativa del director para obtener mejores resultados en modernización de la infraestructura de las instituciones educativas públicas de la RED No 07 de la zona de Nueva Esperanza de Villa María del Triunfo, provincia y región Lima.

CUARTA. - Se recomienda que se mejore la gestión administrativa del director para 
obtener mejores resultados modernización de la maquinaria de las instituciones educativas públicas de la RED No 07 de la zona de Nueva Esperanza de Villa María del Triunfo, provincia y región Lima.

\section{REFERENCIAS.}

ALVAREZ, M. (2006) El Equipo Directivo. Recursos Técnicos de Gestión”, Editora Popular, Primera Edición. Madrid.

NEIROTTI, N. \& POGGI, M. (2004)

Alianzas e innovaciones en proyectos de desarrollo educativo local. IIPE - UNESCO. Buenos Aires.

CARBONELL, J. (2001) La aventura de innovar. El cambio en la escuela. Ediciones Morata, S. L. Madrid.

CHIAVENATO, I. (2007) Introducción a la Teoría General de la Administración, 5ta edición, Editora McGrawhill INTERAMERICANA S.A. Bogotá

MACMILLAN, R.B. (2000) Leadership Succession, Cultures of Teaching and Educational Change, in: $\mathrm{N}$.
Bascia \& A. Hargreaves (Eds) The Sharp Edge of Educational Change. Teaching, leading and the realities of reform. London.

NEIROTTI, N. \& POGGI, M. (2004) Alianzas e innovaciones en proyectos de desarrollo educativo local, IIPE - UNESCO. Buenos Aires.

\section{CÁCERES}

CASAVERDE,

ANTONIA.2008. "La gestión administrativa del director y su influencia en la prestación del servicio educativo en las instituciones educativas públicas de educación primaria de los asentamientos humanos de Dulanto, Santa Rosa y Gambetta del Callao, en el año 2008". Tesis para optar el grado de Magister en Gestión Educativa de la Educación en la Universidad de Educación Enrique Guzmán y Valle. Lima, Perú.

TORRES, R.M. (2000) Reformadores y docentes: el cambio educativo atrapado entre dos lógicas. Los docentes, protagonistas del cambio educativo. Convenio Andrés Bello/Cooperativa del Ministerio de Colombia. Bogotá. 